\title{
Acidic polymer containing sulfunic acid and carboxylic acid groups heterogenized with natural clay: A novel metal free and heterogeneous catalyst for acid-catalyzed reactions
}

\author{
Samahe Sadjadi ${ }^{1}$ MaryamAkbari $^{2}$, Fatemeh Ghoreyshi Kahangi ${ }^{3}$, Majid M.Heravi $^{2}$ \\ 1- Gas Conversion Department, Faculty of Petrochemicals, Iran Polymer and Petrochemicals Institute, \\ Tehran, Iran \\ 2- Department of Chemistry, School of Science, Alzahra University, Vanak, Tehran, Iran \\ 3- Department of Chemistry, University Campus 2, University of Guilan, Rasht, Iran
}

\begin{abstract}
A novel metal-free solid acid catalyst, PHSA, has been designed and synthesized through facile and environmentally benign procedure via using halloysite nanoclay as a natural and biocompatible support. The synthetic protocol included co-polymerization of 2-acrylamido-2-methylpropane sulfonic acid and acrylic acid in the presence of vinyl functionalized halloysite. The metal-free solid catalyst that contained both carboxylic acid and sulfonic acid functionality exhibited high catalytic activity for catalyzing both Knoevenagel condensation and Xanthenes synthesis under mild reaction conditions in aqueous media. The presence of halloysite in the structure of PHSA rendered the catalyst heterogeneous and facilitated its recovery and recyclability compared to the halloysite-free homogeneous counterpart. High catalytic activity and recyclability of the catalyst, simplicity of the preparation procedure, using available and natural clay as a support are the merits of this new metalfree solid acid catalyst.
\end{abstract}

Keywords: Halloysite, Polymer, Metal free catalyst, Knoevenagel, condensation, Xanthenes 